\title{
Failure modes of steel angles connected by one leg
}

\author{
E. Bernatowska \& L. Ślęczka \\ Rzeszów University of Technology, Rzeszów, Poland
}

\begin{abstract}
Paper presents a numerical model, developed to capture ultimate tensile resistance of steel angles connected by one leg using single row of bolts, based on plastic initiation and progression of fracture. The porous metal plasticity was implemented using GursonTvergaard-Needelman material model. Due to such approach, damage of the material microstructure was taken into account. Hierarchical validation of computational model was carried out on the base of own experimental tests. The paper describes the impact of individual geometrical parameters on the global behavior of the steel angle joints and their resistance. Eighty-eight numerical models and experimentally tested specimens gave insight into stress distribution, mechanism of failure and ultimate resistance. Based on these results three different failure modes were distinguished: block tearing, net section tearing and mixed mode.
\end{abstract}

\section{INTRODUCTION}

Steel angle members are very often used in engineering structures. Due to the easiness of manufacture and assembly, the most common form of their joining with construction is connecting only one leg, by single row of bolts. In the case of tension lap joints, category A, their tensile resistance is reached by one of the four forms of failure (EN 1993-1-8 2005): shear of bolts, bearing of bolts, block tearing of angle or failure of angle cross-section (reaching yield strength in gross cross-section, which is called plastic resistance or rupture of net crosssection, called ultimate resistance). The first two resistances are related to the fasteners; the others are related to the load capacity of the angle member.

If the elements in tension are connected only with a certain part of their cross-section, like angle members connected by only one leg, the possible eccentricities can influence the stress distribution in the vicinity of the connection and thus the resistance of the cross-section. The uneven distribution of stresses in the connection area (in the element that is nominally axially tensioned) is called shear lag effect. This phenomenon significantly reduces angle load capacity, Chesson \& Munse (1963), Kulak \& Wu (1997), and should be taken into account when determining the ultimate tensile resistance of sections fastened in this way. In most guidelines such influence is taken into account by means of certain reduction factor by which the net cross-sectional area is multiplied to form the effective cross-section. In this way, the time-consuming determination of additional bending effects is omitted and the entire section is treated as axially loaded.

The necessity to check the block tearing in the section was introduced at the end of the 20th century, Birkemoe \& Gilmor (1978). This form of failure occurs by simultaneous failure in shear at the row of bolts along the shear face of the holes group accompanied by tensile rupture along the line of bolt holes on the tension face of the bolt group.

Paper presents results of parametric analyzes conducted on the base of numerical simulations, carried out on tensile lap bolted connections with angles members. The aim of analyses was to obtain tensile resistances of connections and forms of angles failure. The study was aimed at observing block tearing and rupture of net cross-section of angles. 


\section{DESCRIPTION OF FINITE ELEMENT MODEL}

\subsection{Material model}

The material characteristics used in simulations were obtained from tensile tests of standard coupons cut from angles legs made of steel with nominal grade S275. Medium values of yield stress $f_{\mathrm{y}}$ was $288 \mathrm{MPa}$, and medium value of ultimate stress $f_{\mathrm{u}}$ was $425 \mathrm{MPa}$.

Block tearing and ultimate resistance of angle net cross-section are based on rupture of the material. To capture initiation, progression and final fracture of steel angles, it was decided to use Gurson-Tvergaard-Needleman (GTN) porous material model.

The GTN material model takes into account the influence of microstructural damage on the load capacity and material strength. Damage of the microstructure occurs in the form of voids, which are initiated on the inclusions present in the material. The destruction process takes place through the growth and merging of voids through localized plastic deformation, which is conducted in Kossakowski (2010), Kossakowski \& Wciślik (2018). Full description of conducted material model and its validation is described in Bernatowska \& Ślęczka (2019), Bernatowska (2020).

\subsection{Geometry and model assumptions}

All employed FE models were developed using the package ABAQUS. Finite element model of angle connection included four components: angle, gusset plate, bolt with nut and washers. Only half of the angle specimens were modelled (Figure 1). To model real boundary conditions some part of the gusset plate had blocked displacement in $x$ and $y$ direction. In $z$-direction the load in the form of displacement was applied to the gusset plate. Because of the connection symmetry the end of angle could not move in $z$-direction. Initially both washers and bolt were located concentrically with holes in angle and gusset plate.

Multilinear elastic-plastic material model based on coupon-test results was implemented for gusset plate and bolts (class 10.9). Because all analyzed connections were designed to cause angle failure, porous material characteristics (GTN) were applied only to angle member. Implementation of GTN material model required carrying Dynamic Explicit analysis. Finite elements mesh of angle, gusset plates and washers consisted of three-dimensional, hexahedral 8-node linear bricks with reduced integration (C3D8R). Bolts were built by using C3D8T and C3D6T elements, which are respectively 8-node thermally, coupled bricks with trilinear displacement and temperature and 6-node thermally coupled triangular prism used to complete the mesh. The type of finite elements on the bolt resulted from the temperature load on the bolt shank. The vertical thermal deformation method was utilized, Kim et al. (2007), to apply small clamping force starting from snug-tightened bolts. Shank was modelled as smooth cylinder without thread. Contact between surfaces was defined using general contact option. The frictional effects between surfaces were also included by incorporating the classical isotropic Coulomb friction model in the contact definition, with a friction coefficient $\mu$ equal to 0.1 .

a)

b)

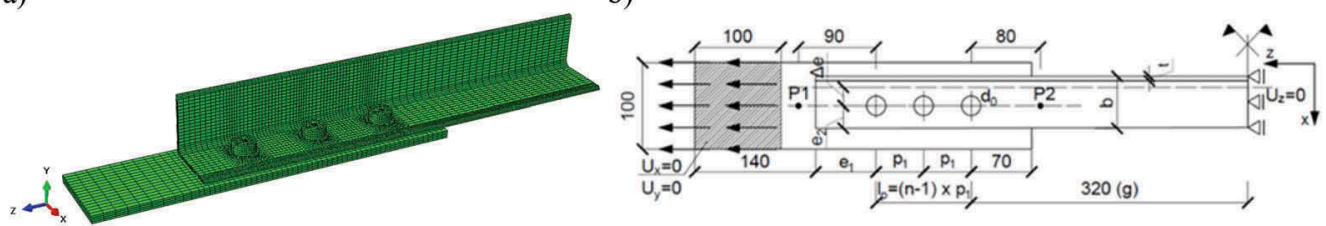

Figure 1. Example of angle member connected by one leg: a) FE model, b) geometry and boundary conditions. 


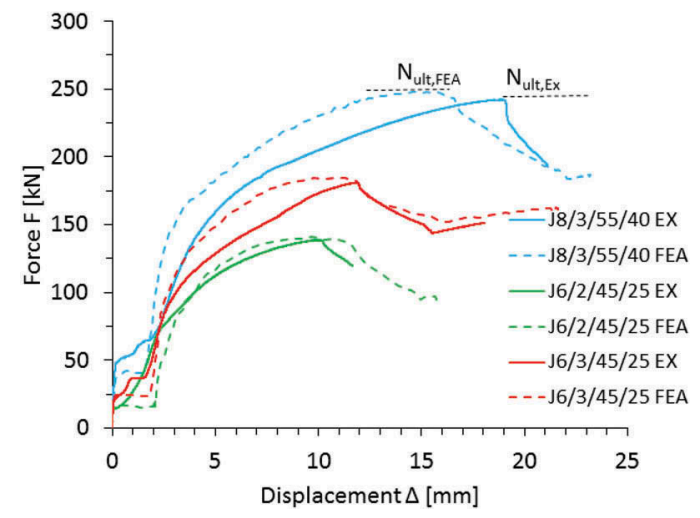

Figure 2. Comparison of force-displacement curves from FE analyses and from experimental research.

The pictures below (Figure 1) present the view on complete model with finite elements mesh and applied boundary conditions, which were used in parametric analyzes.

FE model was validated by comparing the computational results to own experimental data. More information about conducted model validation and verification process is described in publication Bernatowska (2020). Comparison of the computed $F-\Delta$ curves with these from experimental research is depicted in Figure 2 for few of the specimens. Very good accuracy of the failure load prediction was observed. Mean value of $N_{\text {ult,FEA }} / N_{\text {ult,Ex }}$ ratio is equal to 1.02.

\section{PARAMETRIC ANALYSES}

\subsection{Scope of analyses}

To check the influence of individual geometrical parameters on the global behavior of the joints their tensile resistance and forms of failure, parametric analyzes were carried out. Eighty-eight numerical models were built. They were divided into 8 groups, where following parameters were examined:

- the ratio of the width of the leg to its thickness $b / t$ (slenderness of the angle walls), in equal leg angles (A1),

- the spacing between centres of fasteners in a line in the direction of load transfer $p_{1}$ and the total length of the connection $l_{\mathrm{p}}(\mathrm{A} 2)$,

- the edge distance from the centre of a fastener hole to the adjacent edge of angle, measured at right angles to the direction of load transfer $e_{2}$ (A3),

- the end distance from the centre of a fastener hole to the adjacent end of angle, measured in the direction of load transfer $e_{1}$ (A4),

- profile arrangement in connection: joining with a wider or narrower leg in the case of unequal angles (A5),

- angle length (A6),

- profile doubling: single angles vs double angles (A7),

- differentiation of mechanical parameters of steel: elongation and proportion of $f_{\mathrm{u}} / f_{\mathrm{y}}(\mathrm{A} 8)$.

Marked points P1 and P2 were used to obtain longitudinal displacement of connection. Number of bolts vary from 1 to 5. Diameter of used bolts was M18 or M20 respectively for connection with one and more than one bolt. The sizes of angles used in simulations are produced in the steel mills, but several cross-sections were created only for the purpose of analysis, to obtain extreme geometrical proportions of both the cross-section and the connection. Thanks to this "model similarity" was achieved to the entire population of angle profiles of various sizes, connected with bolts with different diameters. 


\subsection{Failure modes obtained from analyses}

The results obtained from the analyzes include:

- force-displacement curves $F-\Delta$ (Figure 2), where $F$ is transmitted force, and $\Delta$ is difference in longitudinal displacements of points P1 and P2 (Figure 1),

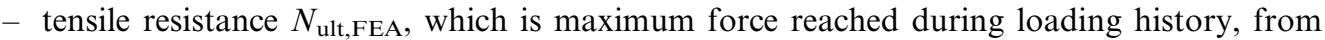
force-displacement $F$ - $\Delta$ curve (Figure 2),

- distribution of effective stresses $\sigma_{\text {eff }}$ (according to Huber-Mises-Hencky hypothesis) for the failure load $N_{\text {ult,FEA }}$ in the sections under block tearing along two paths. Their definitions are presented in Figure 3.

During analyses three failure modes were distinguish (Figure 4): block tearing (marked by symbol BT), net section tearing (symbol NT) and mixed failure mode (symbol M). The main premise for determining the failure mode was the effective stress distribution (for the failure load level $N_{\text {ult,FEA }}$ ) along the section subjected to block tearing (Figure 3). Elements where effective stresses $\sigma_{\text {eff }}$ along F-M or F'-J' achieved or exceeded value $f_{\mathrm{y}}$ were classified as block tearing failure mode (Figure 5a). If stresses along this path (between bolts holes) did not reach yield point, failure mode was net section tearing (Figure 5b). If none of these criteria was met (especially when effective stresses $\sigma_{\text {eff }}$ did not reach $f_{\mathrm{y}}$ in the region of end distance $e_{1}$ ), the element was classified into a mixed failure mode, Figure 5c. Force-displacement curves also confirm these types of failure. For angles, where net-section tearing appeared all $F-\Delta$ curves have des-

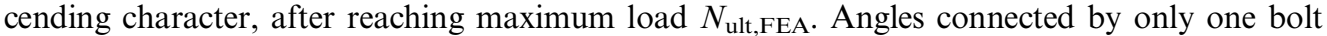
were treated as a separate case and the forms of failure were not distinguished as above.

The length of the connection turned out to have a substantial influence on the tension resistance of the angle. It also influenced the form of failure, i.e. in shorter joints or with a small spacing of bolts $p_{1}$, block tearing or a mixed form was observed, in longer joints (where the bolt spacing was significant) - net section tearing appeared.

a)

b)

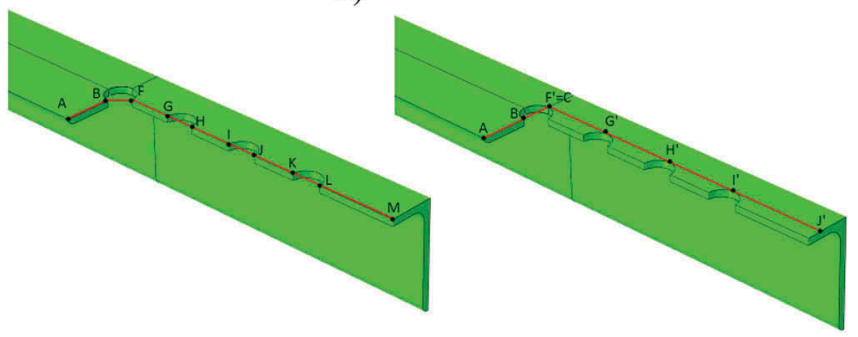

Figure 3. Paths definition for which stresses distribution were obtained: a) in net area subjected to shear, b) in gross area subjected to shear.

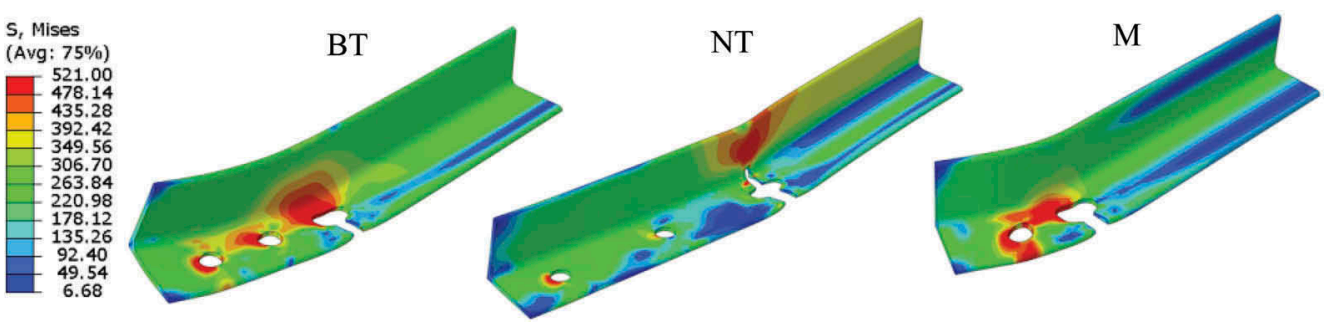

Figure 4. Effective stress distribution and fracture shape in angle members after reaching failure load $N_{\text {ult,FEA }}$ for three failure modes: block tearing (BT), net section tearing (NT) and mixed mode (M). 
a)
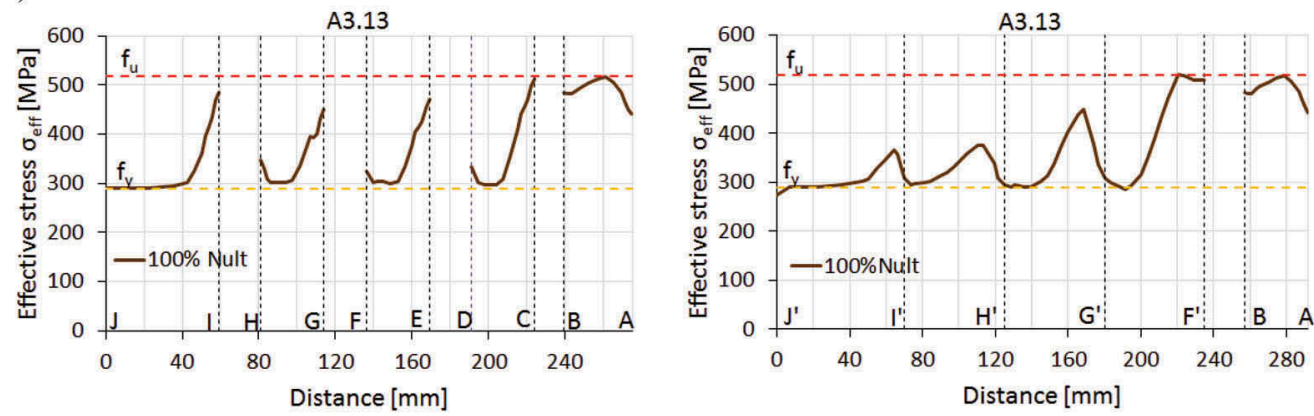

b)
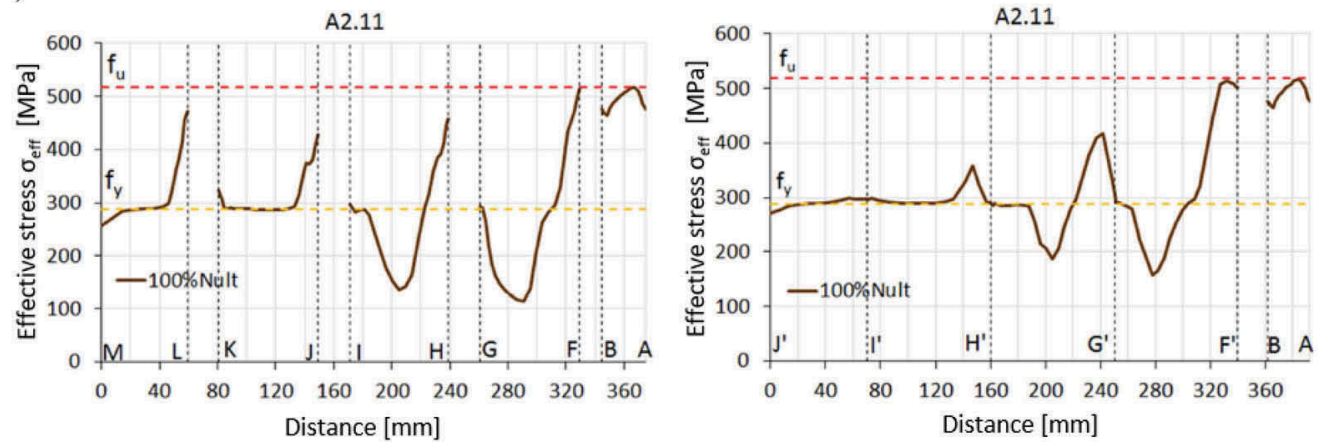

c)
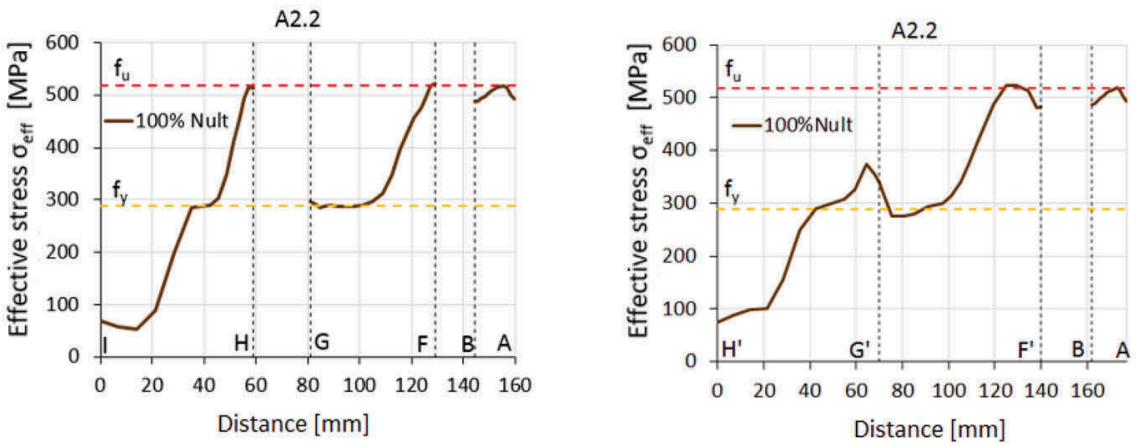

Figure 5. Distribution of effective stresses $\sigma_{\text {eff }}$ for the failure load $N_{\text {ult,FEA }}$ (left diagram shows distribution along net tension and net shear area; right one shows distribution along net tension and gross shear area); a) - c) description in the text.

The distance of the hole axis from the edge of the angle section in the direction perpendicular to the acting force $e_{2}$ (which also determine the eccentricity of the acting load $\Delta e$ ) also turned out to be important. The smaller the value of the eccentricity was, the higher load capacity angle achieved. Mixed failure mode appeared in specimens where $e_{2}<b / 2$ (except long connections with significant bolt spacing).

The analyzes indicated that the influence of unequal angles arrangement was more important for elements where block tearing appeared. For these cases greater tensile resistance can be achieved by connecting angle with a wider leg (a greater area of the tension face $A_{\mathrm{nt}}$ can be obtained). For the net section failure, the width of the connected leg has no influence, but the connection length and the load eccentricity $\Delta e$.

Elements with longer connections are sensitive to changes in the mechanical parameters of steel, which may cause a change in the form of failure: from block tearing (at S235) to net cross-section tearing (at S450). 


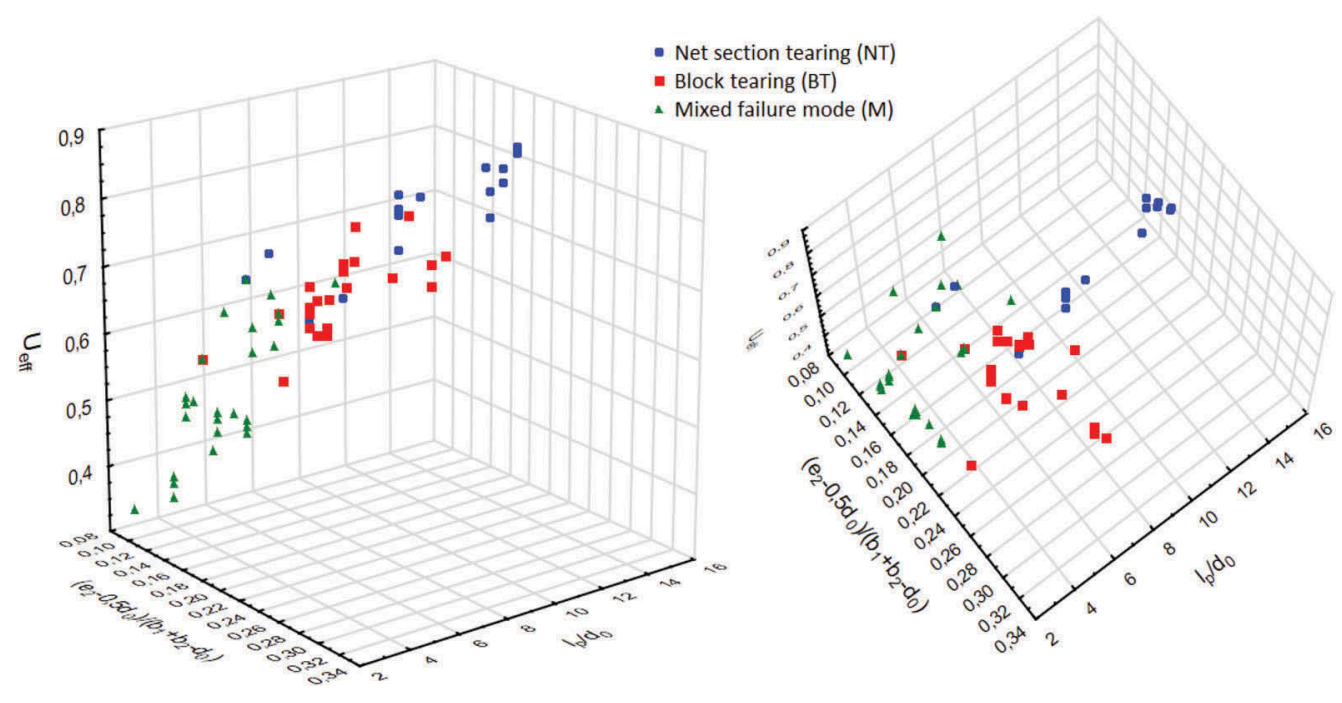

Figure 6. Relative tensile resistance of angles as a function of length of the joints and position of bolt hole measured at right angles to the direction of load transfer.

Comparison of obtained relative tensile resistance $U_{\text {eff }}=N_{\mathrm{ult}, \mathrm{FEA}} /\left(A_{\text {net }} \cdot f_{u}\right)$ for all specimens are shown in Figure $6\left(N_{\text {ult,FEA }}\right.$ is maximum force reached during loading history, $A_{n e t}$ is the net area of the angle, and $f_{u}$ is ultimate strength of the steel). Variable factors in Figure 6 are relative length of the joints $\left(l_{p} / d_{0}\right)$ and relative position of the bolt hole measured at right angles to the direction of load transfer $\left(e_{2}-0,5 d_{0}\right) /\left(b_{1}+b_{2}-d_{0}\right)$, where $l_{p}$ is the distance between the centres of the end fasteners in a joint, $d_{0}$ is diameter of bolt hole, $e_{2}$ is the edge distance from the centre of a fastener hole to the adjacent edge, measured at right angles to the direction of load transfer and $b_{1}$ and $b_{2}$ are widths of angle legs (see also Figure 1).

Slight differences in the obtained resistances in each groups (BT, NT and M) are visible. Specimens in which net section tearing (NT) was observed have net cross-sectional efficiency factor $U_{\text {eff }}$ in the range of $0.68-0.86$. In case of specimens with block tearing failure mode (BT) factor $U_{\text {eff }}$ is in the range 0.60-0.83, and for mixed form of failure $U_{\text {eff }}=0.30-0.70$. Angles with only one bolt, which are not included on diagram below, achieved $U_{\text {eff }}$ from 0.29 to 0.43 .

\section{SUMMARY AND CONCLUSIONS}

During the experimental research it is difficult to distinguish between the block tearing and net cross-section tearing in single angle in tension connected by one leg. In both cases initial form of failure is appearing as a crack forming in tension net cross-section area.

Validated and verified computational FE models based on microstructural damage material modelling give possibility to follow the failure process. Obtained results will be used to calibrate theoretical models for both forms of failure and to find borders between them.

\section{REFERENCES}

Bernatowska, E. \& Ślęczka, L. 2019. Net section fracture assessment of steel bolted joints with shear lag effect. Matec Web of Conferences 262 (09002).

Bernatowska, E. 2020. Numerical simulations of ductile fracture in steel angle tension members connected with bolts. Civil and Environmental Engineering Reports 2 (30): 32-54. 
Birkemoe, P. C. \& Gilmor, M. I. 1978. Behavior of Bearing Critical Double-Angle Beam Connections, Engineering Journal, AISC, Vol. 15: 109-115.

Chesson, E. \& Munse, W.H. 1963. Riveted and bolted joints. Journal of the Structural Division 89 (1): $67-126$.

EN 1993-1-8:2005: Eurocode 3 - Design of steel structures - part 1-8: Design of joints. CEN, Brussels.

Kim, J. \& Yoon, J. \& Kang, B. 2007. Finite element analysis and modeling of structure with bolted joints. Applied Mathematical Modelling 31: 895-911.

Kossakowski, P. 2010. An analysis of the load-carrying capacity of elements subjected to complex stress states with a focus on the microstructural failure. Archives of Civil and Mechanical Engineering 10 (2): $15-39$.

Kossakowski, P. \& Wciślik, W. 2018. Numerical simulation of material damage for structural steels S235JR and S355J2G3. Advances in Computational Design 3 (2): 133-146.

Kulak, G.L. \& Wu, E.Y. 1997. Shear lag in bolted angle tension members. Journal of Structural Engineering 123: 1144-1152. 